\title{
What is in a Viral Stock: Perspectives and Current Limitations of Flow Virometry
}

\author{
Timothy K. Soh ${ }^{1,2,3,4}$ and Jens B. Bosse ${ }^{1,2,3,4, *}$ \\ 1 Centre for Structural Systems Biology, Hamburg, Germany \\ 2 Hannover Medical School, Institute of Virology, Hannover, Germany \\ 3 Cluster of Excellence RESIST (EXC 2155), Hannover Medical School, Hannover, Germany \\ 4 Heinrich-Pette-Institute Leibniz-Institute for Experimental Virology, Hamburg, Germany \\ * Correspondence: jens.bosse@cssb-hamburg.de; Tel.: +49-040-8998-87645
}

\begin{abstract}
:
Herpesviruses produce a plethora of pleomorphic and heterogeneous particle populations. The composition and biological role of these is not understood. Detailed analysis has been challenging due to the lack of multidimensional identification and purification methodologies. Fluorescenceactivated cell sorting (FACS), originally developed to sort objects with at least ten thousand-fold larger volumes, has recently been applied to cellular exosomes as well as viral particles and has been dubbed nanoscale flow cytometry or "flow virometry". In comparison to other nanoparticles, herpesvirus concentrations can be measured with high precision using simple culturing methods. Here, we used this unique capability to evaluate a standard FACS sorter. We demonstrate that detection and separation capabilities were insufficient to distinguish infectious fluorescent viral populations from populations lacking fluorescence and infectivity. Moreover, fluorescent populations did not contain single virus particles but mostly aggregates. On top, analysis of viral samples by flow cytometry was confounded by swarm detection, as multiple objects are measured simultaneously and interpreted as a single object. Despite these technical difficulties, comparison of crude supernatant to gradient purified HCMV revealed that infectious virus is a minor proportion of the particles released from infected cells. Our data stress the need for a set of standardized controls and protocols when applying FACS to biological nanoparticles and highlights technical challenges that need to be solved before flow virometry can achieve its full potential.
\end{abstract}

Keywords: HSV-1; HCMV; flow virometry; extracellular vesicles

\section{Introduction}

A viral infection is propagated through the transmission of infectious particles. For some viruses, these virions are homogenous icosahedral capsids, e.g. poliovirus. In contrast, viruses such as herpesviruses produce both pleomorphic and heterogeneous particles. A herpesvirus particle consists of a capsid surrounded by an amorphous protein layer, which is called the tegument, and wrapped in a membrane. The nebulous tegument of herpesviruses leads to a variable diameter of 200-300 nm. Herpesvirus-infected cells also produce multiple types of particles, some of which are not sufficient for replication. Herpes simplex virus-1 (HSV-1) makes light particles, which contain tegument proteins but no capsid. Human cytomegalovirus (HCMV) releases dense bodies, which lack a capsid, and non-infectious enveloped particles (NIEP), which contain a capsid but no DNA. Often, infection studies are done with little characterized crude supernatant stocks that contain all of the particles released from infected cells. There are currently few methodologies to purify specific components from these crude mixtures, with density-gradient purification being the most widely used [1]. Still, different gradient media with similar density ranges give widely varying results, the viral bands are not sharp, and fluorescence microscopy indicates that selective enrichment of populations is often limited. Therefore, more sensitive methods are needed to better analyze the 
composition of viral stocks and understand the breadth of compositional differences of these pleomorphic viral particles.

Flow cytometry, originally developed to analyze blood cells, has been recently applied as a multidimensional analysis tool to sub-micron objects, termed nanoscale flow cytometry (NFC). The combination of flow cytometry analysis with sorting leads to fluorescence-activated cell sorting (FACS). The application of these technologies to viruses has been called "flow virometry" [2]. Objects must be fluorescently labelled in order to be detected by this machine. While a fluorescent membrane dye will label viruses, it also labels extracellular vesicles (EV) [3]. Therefore, additional criteria are required to correctly identify virions with a flow cytometer, for example, through the fusion of a fluorescent proteins to a viral structural protein. This has been demonstrated for several systems, such as the packaging of Nipah glycoproteins in virus-like-particles (VLPs) [4], HSV-1 tegument proteins into viruses concentrated by ultracentrifugation [5], and the murine leukemia virus (MLV) env protein into particles [6]. Alternatively, antibodies against a viral protein can identify viral proteins on the surface of released particles $[7,8]$. To further characterize the particles released from infected cells, nucleic acid dyes can be added to discriminate between particles that have not packaged the genome [7, 9], but this is method does not work for all viruses, such as for MLV [10]. These studies have led to conclusions concerning viral assembly and the types of particles released from infected cells.

In this work, we aimed to use a standard commercially available BD FACS Aria Fusion to characterize crude and purified herpesvirus stocks and enrich them for proteomic analysis. However, our results demonstrate that the approach is not without complications. We show that our FACS machine failed to detect individual herpesvirus particles that were labelled with various genetically encoded fluorescent tags, could only measure aggregates, and encountered "swarm detection", which is the simultaneous measurement of multiple objects, at moderate viral concentrations. Titration of sorted seemingly pure HSV-1 populations from mixtures revealed that enrichment was minimal. In addition, HCMV infectivity in the sorted positive and negative gates were almost identical, arguing that no specificity could be achieved. Analyzing sorted populations by fluorescence microscopy indicated that mostly aggregates were selected, underlining the lack of single-particle sensitivity. Moreover, we failed to find sideward scatter (SSC) or forward scatter (FSC) specificity for herpesvirus particles and recommend using fluorescence, as has been reported for other viruses [28235684]. We conclude that while the analysis of viral samples by flow virometry has many potential uses, stringent controls are necessary as well as further technological improvements.

\section{Results}

\subsection{Swarm detection convolutes the analysis of nanometer particles}

Our goal was to establish a fluorescence-activated cell sorting (FACS) workflow to characterize and isolate defined herpesvirus populations from crude stocks. To this end, we first assessed if the available FACS machine was able to detect sub-micron sized beads. Analysis of a commercial standard demonstrated the used machine's ability to distinguish beads down to $160 \mathrm{~nm}$ by SSC (Figure 1A).

Next, we tested if the machine measured only single particles or encountered swarm detection, the simultaneous measurement of multiple objects. If only one object is being detected in every event, a serial dilution series will show a linearly decreasing curve of detected particle numbers. However, if multiple objects are detected in a single event with a random distribution, the dilution series will follow a Poisson distribution (Figure 1B). As shown in Figure 1C, a dilution series of beads fit a Poisson distribution, which is indicative of swarming.

By mixing fluorescent beads of two different colors, red and yellow, we could also test for swarming by examining the events' fluorescence. The presence of double-positive red and yellow events confirmed swarm detection at high concentrations. The loss of the double-positive population by dilution demonstrated that these are due to coincident events, not aggregates. 

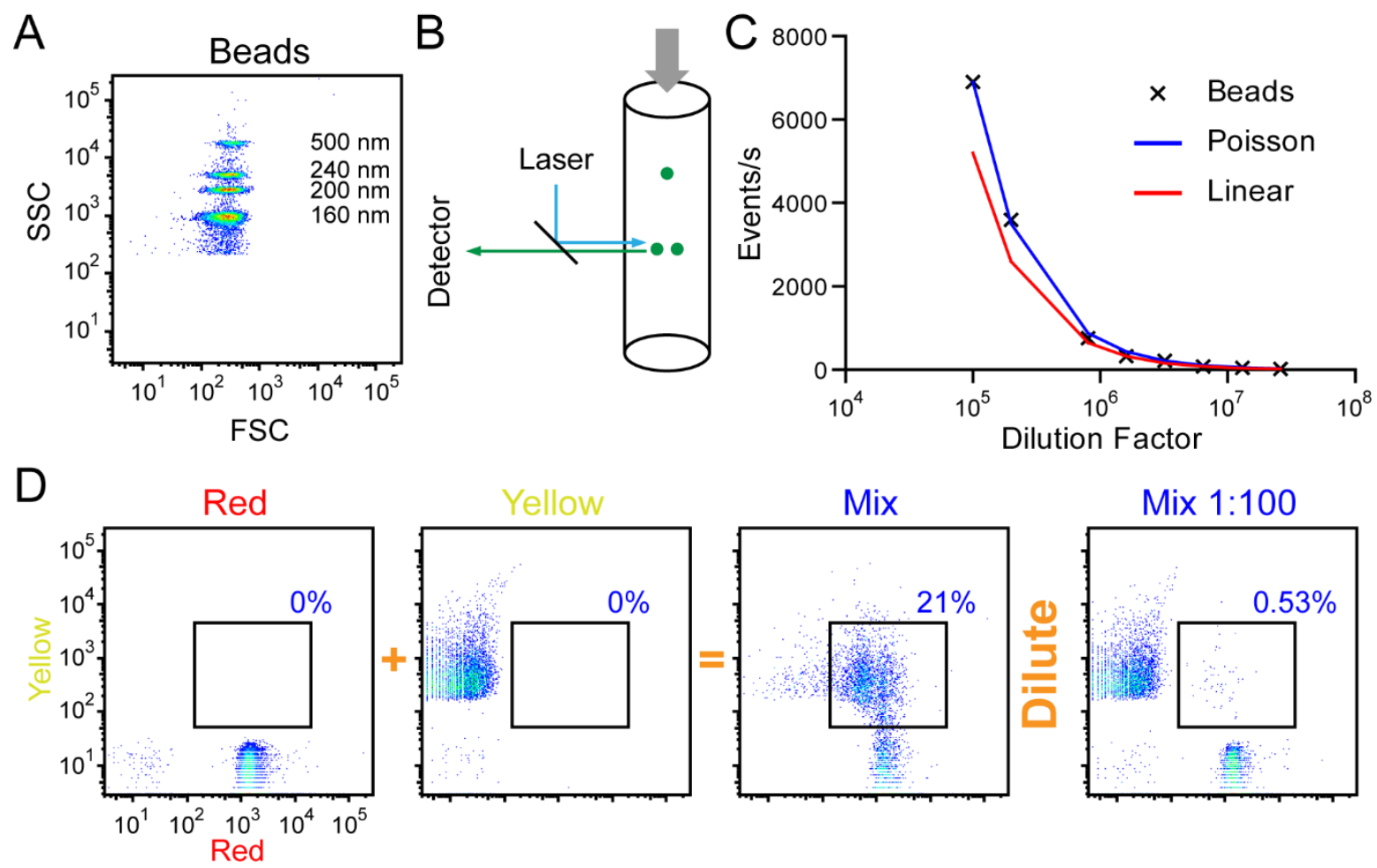

Figure 1 Swarm detection of fluorescent beads. (a) The sensitivity of a BD FACS Aria Fusion was tested with calibration beads of indicated diameter. (b) Flow cytometers measure objects that pass through a capillary. If two objects pass simultaneously, they will be measured as a single object known as swarm detection. (c) Red and yellow fluorescent beads were mixed 1:1 and serially diluted for analysis by flow cytometry. The number of events in $10 \mathrm{~s}$ was measured and plotted. A model assuming a Poisson or linear distribution is plotted based on the highest dilution, $1: 2.6 \times 10^{7}$. (d) Red beads (Red) and yellow beads (Yellow) were analyzed separately and after mixing (Mix), as in (c). The mixed sample was also diluted (Mix 1:100) and analyzed again. The percentage of red ${ }^{+}$yellow $^{+}$ double-positive events are labelled.

\subsection{Analysis of herpesvirus particles is affected by swarm detection}

To test whether swarm detection is a concern for flow virometry of herpesviruses, we mixed gradient-purified eGFP- with mCherry-VP26 capsid-tagged HSV-1 [11, 12] (Figure 2A). We looked for double-positive events by flow cytometry (Figure 2B). We found a shift in the populations towards double-positive events. Dilution of this mixture shifted the populations back to where the single-virus samples fell, thus confirming that swarm detection was an issue for herpesviruses. Of note, the mixed sample did not generate a single population; instead, we observed a smear and shifted towards where a double positive event should be. This observation is consistent with swarm detection of larger aggregates that mixed with smaller amounts of the other viral preparation. 
A

HSV-1 HSV-1

VP26-mCherry VP26-eGFP

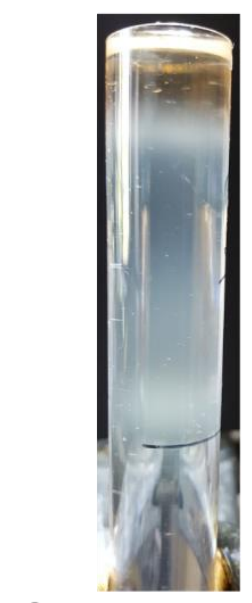

C

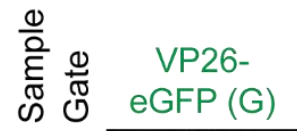

G
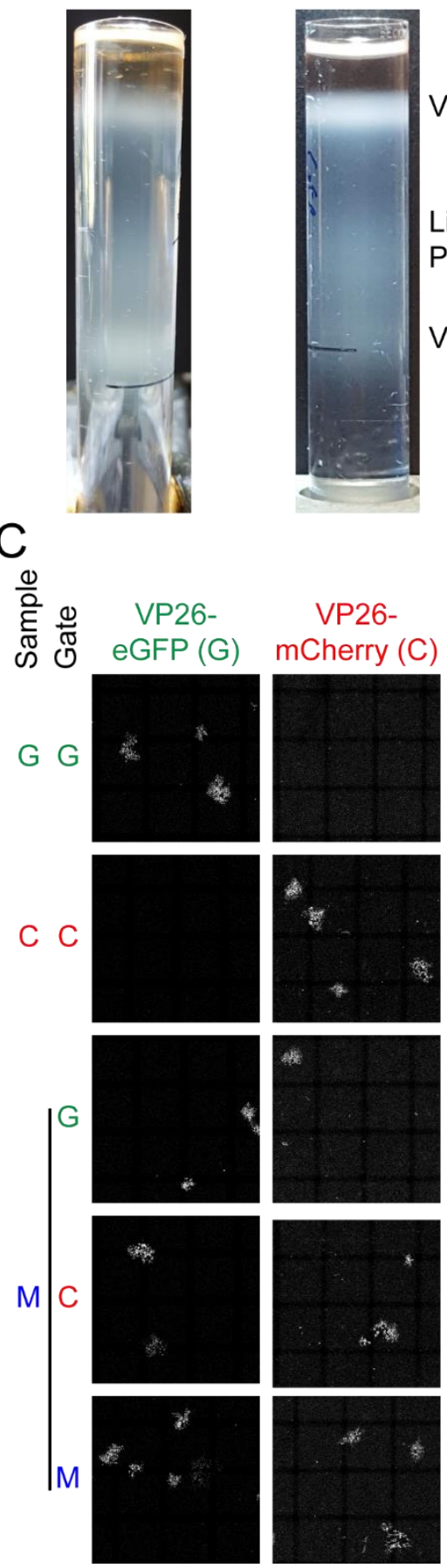

Vesicles

Light

Particles

B

Virus


Figure 2 Single HSV-1 particles are not detected by flow virometry. (a) HSV-1 VP26-eGFP and HSV1 VP26-mCherry were purified on a 5-30\% Optiprep continuous gradient. (b) Flow cytometry analysis of gradient purified HSV-1. HSV-1 VP26-eGFP and HSV-1 VP26-mCherry were diluted to generate the same number of events/s. The viruses were mixed 1:1 to generate the "Mix" sample, where each virus' concentration is the same as in the single samples. "Mix" was diluted 1:10 to generated "Mix 1:10". n=3. (c) Populations sorted by FACS were titered, and plaques were imaged with a fluorescence microscope. Scale bar represents $500 \mu \mathrm{m}$. n=3. (d) The titer of HSV-1 VP26-eGFP and HSV-1 VP26mCherry in each sample in D were counted. The dotted line represents the sensitivity of the assay. $\mathrm{n}=3$. The sample labels are HSV-1 VP26-eGFP (G), HSV-1 VP26-mCherry (C), or the mixture of these two viruses (M). The gate labels are the gate of HSV-1 VP26-eGFP (G), HSV-1 VP26-mCherry (C), or 
where a double positive mixture of these two viruses would theoretically fall (M). The plaque labels are for $\mathrm{GFP}^{+}$plaques $(\mathrm{G})$ or $\mathrm{mCherry}^{+}$plaques $(\mathrm{C})$.

To test if swarming led to physical mixing, we sorted the single and mixed samples for single and mixed events. These fractions were analyzed by plaque assay, a method to quantify the absolute number of infectious viral units, and the number of eGFP and mCherry positive plaques were counted. Consistent with the flow cytometry results, the sorted populations from the unmixed HSV1 VP26 viruses contained only the corresponding virus, and the mixed population from the mixed sample had both viruses (Figure 2C, D). Surprisingly, titration of the sorted single-positive populations from the mixed HSV-1 sample (Figure 2D) demonstrated that the eGFP gate contained infectious HSV-1 VP26-mCherry virus and vice versa. These results support not only the notion that the FACS machine was prone to swarm detection, but more importantly that it was unable to detect single virus particles.

\subsection{Single HCMV virions are not detected}

Since the infectious green virus was present in the red fluorescent HSV-1 fraction and vice versa, we concluded that the FACS machine could not detect single virus particles. One possibility is that the criteria the machine uses to identify an object failed to detect single virions. Flow cytometry registers an event when there is a signal above the specified threshold in the chosen triggering channel (Figure 3A). To test if our incapability to detect single virus events is due to triggering on a low-signal channel, we used an HCMV mutant with two labels (HCMV pp150-eGFP gM-mCherry). pp150 is a tegument protein that binds to the capsid, and gM is a membrane-bound glycoprotein. Objects that contain both fluorophores contain both a capsid and membrane and are likely an intact virion. We used gradient purified HCMV to enrich for infectious virus and remove non-virus signal (Figure 3B).

Typically, SSC is used to trigger events. Since cells are highly granular, this is not a problem with cells. To ensure that virions were not missed, we also triggered events with the minimum threshold value for eGFP and sorted the eGFP positive population. For a negative control, we needed to sort an eGFP negative population. To achieve this, we triggered events on a BuV 737 channel, which showed high autofluorescence under all conditions tested, and sorted the eGFP population with less signal than the minimal threshold, i.e. 200. All three triggering settings showed different populations (Figure 3C). We sorted and titred each population, and surprisingly, all three populations contained about equal amounts of infectious virus in the range of $10^{5}$ plaque-forming units (PFU)/mL. Therefore, we concluded that regardless of which channel is used for triggering, enrichment of infectious virus could not be achieved. Instead, the input virus with a concentration of $10^{8} \mathrm{PFU} / \mathrm{mL}$ was seemingly non-selectively diluted about one-thousand-fold.

Like in the case of HSV-1, HCMV events that were determined as non-fluorescent by the machine contained infectious virus. We compared how much virus entered the machine and how many events were measured to estimate the machine's sensitivity threshold. In the case of HSV-1, the PFU/SSC event ratio was about 30. For HCMV, the PFU/SSC event was about 200, and PFU/GFP event was about 1 . This number has to be multiplied by the number of particles that correspond to a plaque-forming unit (the so-called particle-to-PFU ratio) since not every particle is infectious. This number is about 100 for both HSV-1 [7609061] and HCMV [29743375], which leads us to conclude that the flow cytometer is not detecting the vast majority of particles. 
A


B

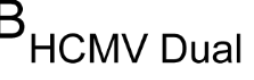

C

Triggered by:
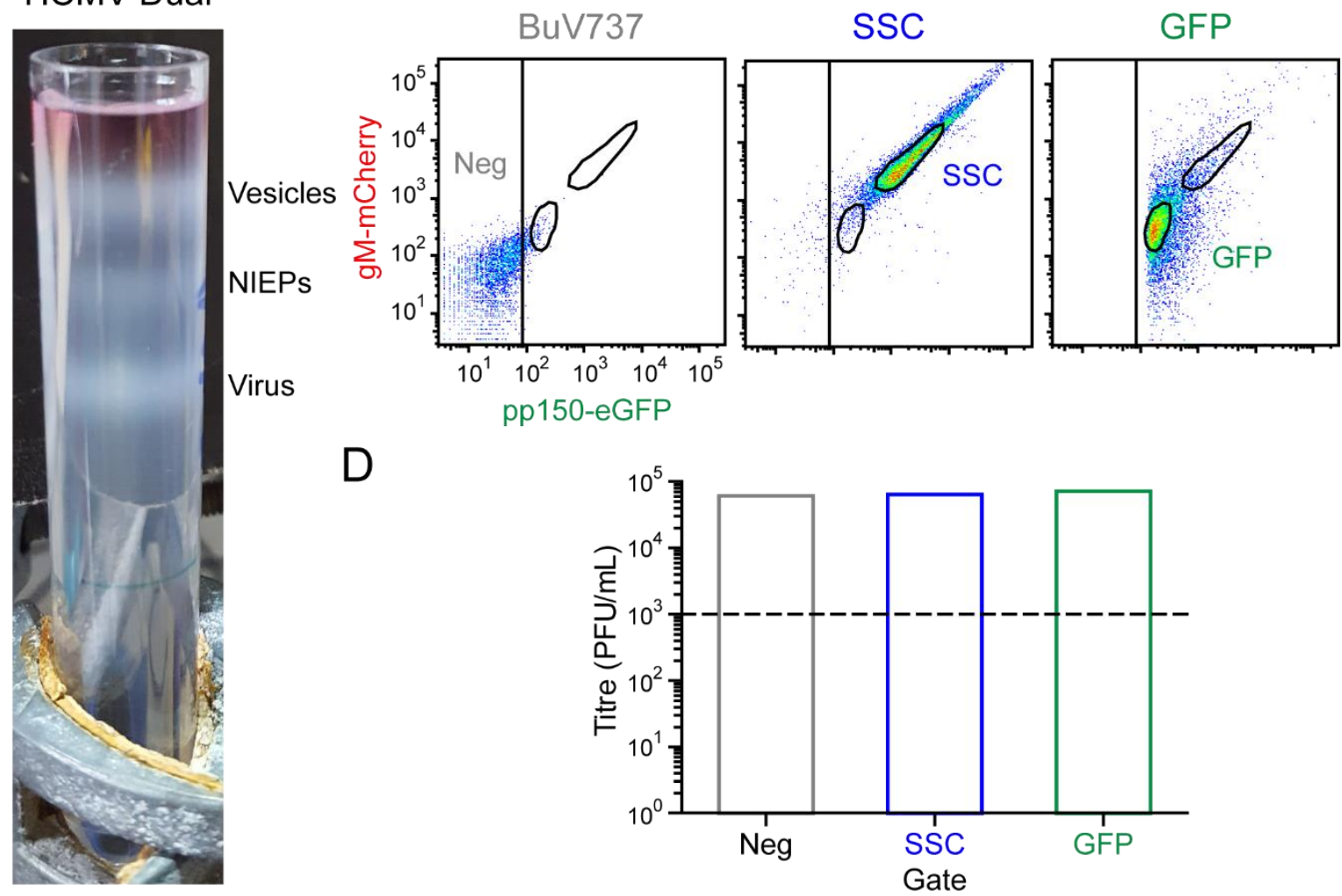

Figure 3 Single HCMV particles are not detected by flow virometry. (a) Flow cytometers define events as when the signal is above a certain threshold. If an object passes through but does not generate enough signal to exceed the threshold, the flow cytometer does not register it as an event. (b) HCMV pp150-eGFP gM-mCherry (Dual) was purified with a continuous 15/35\% tartrate/glycerol gradient to enrich virus from vesicles and non-infectious enveloped particles (NIEPs). (c) Gradient purified virus from B was analyzed by flow cytometry with triggering on different channels, BuV737 (Neg), SSC, or GFP, with the minimum threshold of 200. (d) The gates were sorted and titered. The dotted line represents the sensitivity of the assay.

\subsection{Sorted fractions mainly contain viral aggregates}



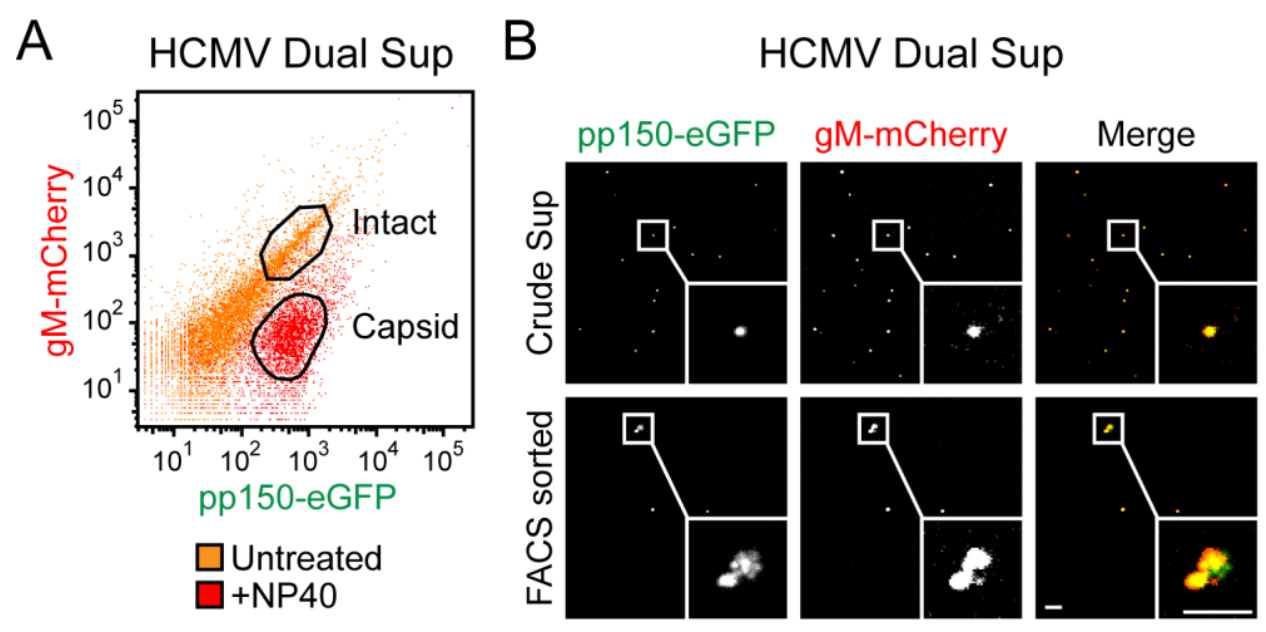

Figure 4 Flow virometry can detect aggregates of fluorescent HCMV. (a) Crude supernatant from HCMV pp150-eGFP gM-mCherry (HCMV Dual Sup) \pm 1\% NP40 was analyzed by flow cytometry. n=3. (b) Fluorescence microscopy images of HCMV pp150-eGFP gM-mCherry crude supernatant before (Crude Sup) and after (FACS sorted) sorting by FACS. Scale bar represents $5 \mu \mathrm{m}$.

Since our results indicated that flow cytometry could not detect single virus particles, we asked what the detected fluorescent events contained. To test whether the objects contained intact virus, we added NP40, a detergent, and reanalyzed the sample. Artifacts such as autofluorescent debris and electrical noise would not be affected by such a treatment. However, NP40 will remove the membrane from virus particles but leave the capsid intact. Therefore if these are virions, the double-positive population should disappear and be replaced with an eGFP-only population. Indeed, this is what we saw, suggesting that the signal is from viral proteins (Figure 4A). Detergent treatment of supernatant also resulted in the loss of the double negative population. This population likely corresponds to the low-density vesicles that are seen during gradient purification (Figure 3B). Detergent treatment dissolved these particles, and therefore they were not detected.

We sorted these populations by FACS and analyzed them with fluorescence microscopy (Figure 4B). While the unsorted crude supernatant displayed symmetrical diffraction-limited objects, which indicate single virus particles, the sorted sample showed aggregates. These data demonstrated that the fluorescent events detected by flow cytometry are not single particles and that sorting actually enriched for aggregates.

\subsection{Supernatant virus is indistinguishable from background events by scattered light}

SSC and FSC are markerless measurements that characterize cells and have been applied to viruses [9]. A comparison of fresh media (negative control) to crude viral supernatant demonstrated that supernatant is nearly identical to the background events found in media (Figure 5A). While a BD FACS Aria Fusion could distinguish beads down to $160 \mathrm{~nm}$ (Figure 1A), the inability to detect $<300$ $\mathrm{nm}$ vesicles and viruses was likely due to membrane-bound vesicles being less reflective and having a lower optical density.

If virus particles are a minor component of the crude supernatant, then perhaps the viral SSC or FSC signal is being masked by the non-viral components. To address this, we gated the media and supernatant populations for eGFP and mCherry, i.e. the viral proteins (Figure 5B). Both samples displayed a single population with the supernatant virus being slightly brighter than the media background population. Comparison of the SSC and FSC of these gated populations revealed the same pattern as observed in Figure 5A; namely, the viral supernatant is identical to media. Comparing the scattered light from viral supernatant with fresh media demonstrated that this measurement parameter cannot identify virus particles. 
A
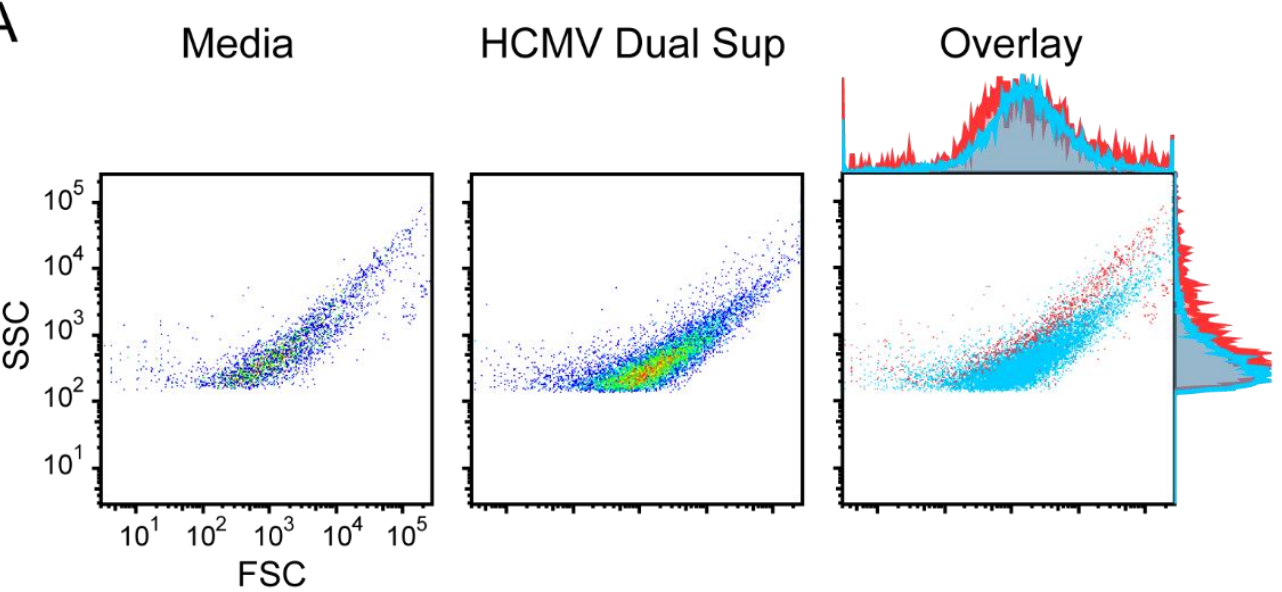

B
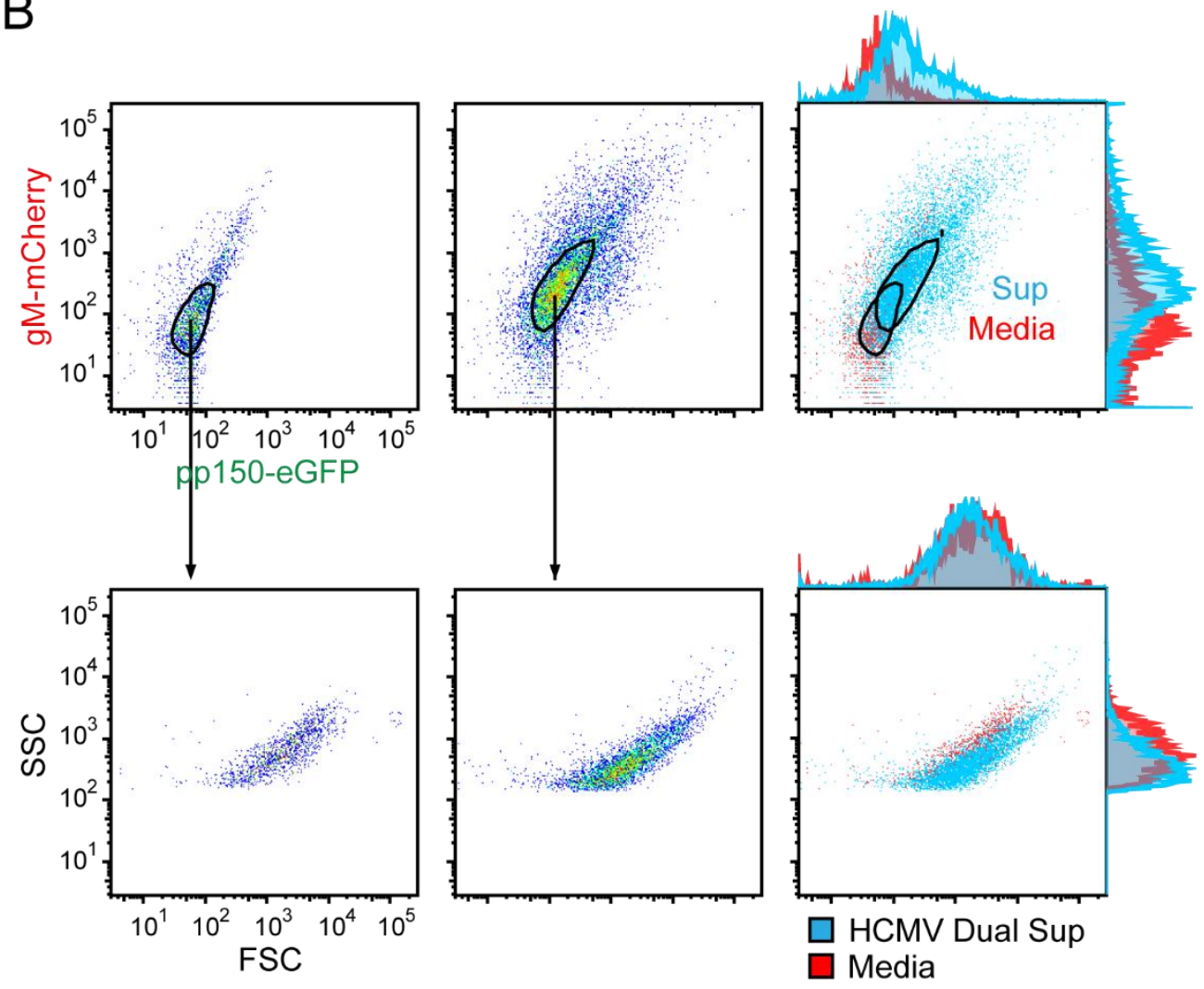

Figure 5 HCMV supernatant is indistinguishable from background by SSC and FSC. (a) Fresh media (Media) and HCMV pp150-eGFP gM-mCherry supernatant (HCMV Dual Sup) were analyzed by flow cytometry for SSC and FSC as well as eGFP and mCherry. (b) The eGFP ${ }^{+}$mCherry ${ }^{+}$viral population was compared against the GFP- mCherry- population from media. The samples were gated based on fluorescence, and these populations were compared by SSC and FSC.

\section{Discussion}

\subsection{What is in a viral stock, and how can we analyze it?}

Propagation of viruses in cell culture is mostly performed through crude stocks. At least for herpesviruses, the contents of these stocks are not well understood. Classically, density gradients are used to purify virus particles to a higher degree through physical characteristics ascribed to infectious virus. However, this is a bulk methodology and thus does not permit single-particle analysis for parameters such as protein composition and occupancy of viral structural proteins that could influence individual particle infectivity. For each infectious unit of most herpesviruses, there are about 100 non-infectious particles [13, 14], and it is currently unclear if or how individual particles 
differ from each other. As proteomic approaches are not sensitive enough to analyze individual particles, a single-particle sorting method would be ideal for enriching similar particles for subsequent analysis. Given the plethora of fluorescent viral mutants [15], a fluorescent approach could take advantage of this library and sort particles into different bins for downstream proteomic analysis.

Therefore, we explored the possibility of using flow cytometry to analyze and sort crude viral stocks for downstream analysis as recent reports suggested the feasibility of such an approach [9]. Unfortunately, we discovered that virus flow cytometry or "flow virometry" with current standard machines is challenging and technical improvements are necessary before the broader applicability of this approach.

\subsection{Flow virometry with different viruses and machines}

This work demonstrates that our standard BD FACS Aria Fusion failed to detect single HSV-1 or HCMV particles. Instead, it detected aggregates and encountered swarm detection even at moderate titres. The key to demonstrating single-particle sensitivity is the lack of virus, as measured by an independent method, in a negative gate. For HIV, ELISA against the capsid protein has been used to measure the virion concentration in sorted populations [16]. In this publication, the capsid concentration was similar between populations with different amounts of a fluorescent structural protein, including the negative population [16]. In contrast, flow virometry of the much larger 300 $\mathrm{nm}$ vaccinia virus shows that the negative population does not contain infectious virus [17]. In addition, the mutually exclusive content of sorted $650-1200 \mathrm{~nm}$ giant viruses is demonstrated by PCR [18]. Perhaps larger viruses are more amenable to flow virometry. We also encountered swarm detection, which is not unique to our system. It is reported for $60 \mathrm{~nm}$-sized dengue virus particles analyzed with an LSRII [3] and $120 \mathrm{~nm}$ HIV particles with an A50Micro [19]. Scattered light was previously reported to identify herpesvirus particles [9]. We analyzed viral samples with this approach and could not discern it from a negative control. The inability to detect virions by light scattering [19] and the advantage of triggering on a fluorescent channel [10] have been reported previously for other viruses. As standard FACS machines are optimized to separate individual cells, which are much larger than most viruses, we believe that choosing the appropriate detection criteria and validating the sorted populations with an independent method is critical for flow virometry.

As a caveat, the presented work used a standard BD FACS Aria Fusion while some studies used modified systems with much stronger lasers, up to 10 -fold $[3,17,19,20]$. While this difference could be a key factor for achieving single-particle sensitivity, it would drastically limit the widespread availability of flow virometry. While some success has been achieved for larger viruses, this may not be possible with all viruses.

\subsection{Herpesvirus infected cells produce multiple types of particles}

Despite the discussed limitations, our results showed that particles isolated by gradient purification were a minor fraction of what is released from cells. These events might be aggregates due to ultracentrifugation, which has been previously reported during flow virometry analysis [19]. However, given that the infectious virus band is not the only band in the gradient, the supernatant contains more species than just virus. In either case, the crude supernatant's main population is absent in the gradient-purified sample (Figure 3C and Figure 4A), which is classically deemed the infectious virus. While the purified population is indeed infectious, the function, or perhaps lack of function, of the other types of particles produced from infected cells has not been firmly established. In HSV-1, light particles, which are particles that do not enclose a capsid and contain only tegument proteins, are also released. While these are not infectious by themselves, they would deliver viral proteins to the target cell, potentially facilitating initiating infection [21]. Given that these non-gradient purified particles are the predominant particles released from cells, at least for HCMV, perhaps they have a biological function. There is evidence for a role of EVs in viral pathogenesis [22, 23]. For HCMV, 
infected cells produce EVs that have incorporated viral proteins [24, 25], and EVs may play a role in viral spread [26]. These non-infectious viral particles may be important for priming cells for infection.

An unexpected observation appears when comparing HSV-1 to HCMV. For both of these herpesviruses, the non-fluorescent population in flow cytometry is infectious. Interestingly, the fluorescent population of HSV-1, but not HCMV, had a higher titre than this "background". Analysis of sorted fluorescent populations by microscopy demonstrated that these were aggregates. Together, this implies that aggregates of HSV-1, but not HCMV, are infectious. HSV-1 is a member of the subfamily Alphaherpesvirinae while HCMV is a member of Betaherpesvirinae, but they are from the same Herpesviridae family. They are structurally similar, and both can enter the cell by fusing with the plasma membrane $[27,28]$. An aggregate of intact virions is expected to still be capable of fusing at the plasma membrane and initiating infection. Perhaps the contents of the aggregates are different between these viruses. Equilibrium density gradient purification selects for objects with a specific average density. HCMV may be more prone to forming aggregates of non-infectious or broken particles.

\subsection{Further development of flow virometry}

Moving forward, it is vital to control flow virometry approaches carefully. Single virion sensitivity and avoidance of swarm detection need to be kept in mind. One possible solution to detecting small particles is by increasing the detection lens' numerical aperture. This could increase the sensitivity of the scattered light and fluorescence channels. New technology, such as the camerabased flow cytometers, show promise for detecting single particles [29, 30], but sorting is not currently commercially available. Another concern is that viruses have many similarities to EVs, which means that correct identification of virus particles from debris and EVs requires multiple markers [10]. Importantly, this means that the EV field has similar concerns, and the standardized protocols to avoid artifacts [31] could be applied. Of note, viruses have an advantage over EVs in that they can be quantified through culturing. This is essentially signal amplification that allows for the detection of a single infectious unit. For many viruses, this is a simple method to determine how much material is put into a flow cytometer and how much comes out.

The limited success of flow virometry provides a glimpse into the complexity of viral productivity and transmission and stresses the need for independent methodological approaches to challenge established virological assays. Flow virometry, especially in combination with proteomic analysis of sorted populations, could take a prominent role in exploring what is actually in a viral stock. However, current technical challenges, as detailed in this report, need to be solved.

\section{Materials and Methods}

\subsection{Cells and viruses}

HFF-1 cells (ATCC cat\# SCRC-1041) were grown in 5\% fetal bovine serum (FBS) superior (Biochrom AG cat\# S0615) $1 \mathrm{ng} / \mathrm{mL}$ recombinant human Fibroblast Growth Factor (Pepro Tech EC GmbH cat\# 100-18B) Dulbecco's Modified Eagle Medium (DMEM) (Gibco cat\# 61965059). Vero-B4 cells (DSMZ ACC 33) were grown in 10\% FBS DMEM. HCMV pp150-eGFP gM-mCherry was a kind gift from Christian Sinzger [32], and HSV-1 VP26-eGFP and HSV-1 VP26-mCherry were kind gifts from Beate Sodeik [11, 12].

\subsection{Plaque assay}

Samples were serially diluted in 10\% FBS DMEM and used to inoculate confluent HFF cells for one hour. After the inoculation, overlay (0.6\% methylcellulose 2\% FBS DMEM) was added to the wells, and the plates were incubated at $37^{\circ} \mathrm{C} 5 \% \mathrm{CO}_{2}$. At 9 dpi, the plates were fixed in $3.75 \%$ formaldehyde (Geyer Th. GmbH \& Co.KG cat\# 252549) in PBS for $15 \mathrm{~min}$ and stored in PBS. Counting was performed with an epifluorescent Olympus IX70 microscope at 40x total magnification with standard GFP and RFP filter cubes. 


\subsection{Gradient purification}

Vero cells in $4 \times 15 \mathrm{~cm}$ plates were infected with HSV-1 at MOI 0.01. At three dpi, the supernatant was collected, and cell debris was removed by centrifugation at 1200xg for 5 min. Virus was concentrated by centrifugation at $14000 \mathrm{xg}$ for $1.5 \mathrm{~h}$ at $4^{\circ} \mathrm{C}$. The pellet was resuspended overnight in $1 \%$ FBS PBS on ice. Before loading the concentrated virus onto the gradient, large aggregates were removed by centrifugation at $18000 \mathrm{xg}$ for $1 \mathrm{~min}$ at $4^{\circ} \mathrm{C}$. Gradient purification was performed with a 5-30\% Optiprep in PBS continuous gradient made with a Gradient Master (BioComp Instruments) for an SW41 rotor. After centrifugation at 18000xg for $1.5 \mathrm{~h}$, the bands were isolated. The virus was diluted 1:2 in PBS before immediate analysis by flow virometry to decrease the sample's viscosity.

HFF cells in a $1 \times 15 \mathrm{~cm}$ plate were infected with HCMV at MOI 0.05. At $7 \mathrm{dpi}$, this plate was trypsinized and split equally into $16 \times 15 \mathrm{~cm}$ plates of HFF cells. At $7 \mathrm{dpi}$, the supernatant was harvested. Virus was concentrated as for HSV-1; namely, the supernatant was clarified by centrifugation at $1200 \mathrm{xg}$ for $5 \mathrm{~min}$, the virus was pelleted at $14000 \mathrm{xg}$ for $1.5 \mathrm{~h}$ at $4^{\circ} \mathrm{C}$ followed by resuspension overnight, and large aggregates were removed by centrifugation at $18000 \mathrm{xg}$ for $1 \mathrm{~min}$ at $4{ }^{\circ} \mathrm{C}$. Gradient purification was performed with a continuous gradient consisting of $15 \%$ sodium tartrate with $30 \%$ glycerol $(\mathrm{w} / \mathrm{w})$ and $35 \%$ sodium tartrate $(\mathrm{w} / \mathrm{w})$ in $40 \mathrm{mM}$ sodium phosphate $\mathrm{pH}$ 7.4. This was made with a Gradient Master (BioComp Instruments) for an SW41 rotor. After centrifugation at $65000 \mathrm{xg}$ for $1.5 \mathrm{~h}$, the bands were isolated and diluted 1:2 in PBS.

\subsection{Flow virometry and fluorescence-activated cell sorting}

Flow cytometry and sorting were performed on a BD FACS Aria Fusion equipped with $20 \mathrm{~mW}$ $355 \mathrm{~nm}, 50 \mathrm{~mW} 488 \mathrm{~nm}$, and $50 \mathrm{~mW} 561 \mathrm{~nm}$ lasers and emission filters 740/40 (BuV737), 525/50 (GFP), and 610/20 (mCherry). The nozzle size was $70 \mu \mathrm{m}$ and BD FACS Diva Software v.8.0.1 was used. SSC and FSC measurements were performed with the $488 \mathrm{~nm}$ laser. Unless otherwise stated, triggering was performed on SSC with a threshold of 200 . Sorting was into $1.5 \mathrm{~mL}$ tubes containing $10 \mu \mathrm{L}$ HFF media. Volumes of input and sorted samples were measured by micropipetting.

Calibration beads (Cosmo Bio Co. LTD cat\# 7803) were used to test the limit of SSC and FSC of the FACS machine. Red (Invitrogen cat\# F8801) and yellow (Invitrogen cat\# F8803) fluorescent beads were used to test the machine for swarming. The beads were mixed 1:1 and serially diluted in PBS. Each dilution was measured for 10000 events and $10 \mathrm{~s}$.

Detergent treated samples were generated by adding a 10x stock to a final concentration of $1 \%$ NP40 (VWR A1694). For gradient purified virus, the virus was first diluted 10-fold in PBS before the addition of NP40 to prevent precipitation from the gradient solute.

\subsection{Imaging fluorescent plaques}

Fixed 24-well plastic plates were imaged by confocal laser scanning microscopy. This was performed with a Nikon Eclipse Ti2 equipped with a Nikon A1 confocal laser scanning unit. Images were acquired with a Plan Apo $\lambda$ 4x air NA 0.2 objective at $1.5 \mu \mathrm{m} /$ pixel and GaAsP detectors. Excitation was performed sequentially with $489 \mathrm{~nm}$ and $561 \mathrm{~nm}$ lasers through a quad filter (405/488/561/640), and emission was measured with bandpass filters 525/50 for eGFP and 595/50 for mCherry.

\subsection{Imaging fluorescent virus}

$\mu$-Slide eight well glass bottom ibidi chambers (cat\# 80827) were precoated with $0.002 \%$ poly-Llysine (Sigma cat\# P4707) diluted in PBS for $5 \mathrm{~min}$ at room temperature. Virus was added and incubated for $5 \mathrm{~min}$ at room temperature. Chambers were washed 3x with PBS before imaging in PBS.

Images were acquired with a spinning disk confocal microscope, which uses a Nikon Eclipse Ti2 body and Yokogawa CSU-W spinning disk with Borealis homogenator. The samples were placed on a Nano-Drive piezo stage (Mad City Labs Inc) and imaged with an Andor iXon Ultra DU-888U3 
EMCCD camera. Samples were excited with a $488 \mathrm{~nm}$ or $561 \mathrm{~nm}$ laser through a quad filter (405/488/568/647) and imaged with a Plan Apo $\lambda$ 100x oil NA 1.45 for a resolution of $131 \mathrm{~nm} /$ pixel.

Author Contributions: Conceptualization, Timothy K. Soh and Jens B. Bosse; Data curation, Timothy K. Soh; Formal analysis, Timothy K. Soh; Funding acquisition, Jens B. Bosse; Investigation, Timothy K. Soh; Methodology, Timothy K. Soh and Jens B. Bosse; Project administration, Jens B. Bosse; Resources, Timothy K. Soh and Jens B. Bosse; Supervision, Jens B. Bosse; Validation, Timothy K. Soh and Jens B. Bosse; Visualization, Timothy K. Soh and Jens B. Bosse; Writing - original draft, Timothy K. Soh and Jens B. Bosse; Writing - review \& editing, Timothy K. Soh and Jens B. Bosse.

Funding: This research was funded by a Wellcome Trust Collaborative Award (209250/Z/17/Z) to JBB. TKS is funded through this grant. The Bosse lab is funded by the Deutsche Forschungsgemeinschaft (DFG, German Research Foundation) under Germany's Excellence Strategy - EXC 2155 "RESIST" - Project ID 390874280 as well as the Heinrich Pette Institute, Leibniz Institute for Experimental Virology.

Acknowledgments: Flow cytometry was performed in the FACS core facility at the HPI. We would like that thank Arne Duesedau and Jana Henessen for their technical and practical expertise and support. We thank Wolfram Brune, Beate Sodeik, Christian Sinzger, and Kerstin Sampaio for their generous gift of reagents and their support.

Conflicts of Interest: The authors declare no conflict of interest. The funders had no role in the design of the study; in the collection, analyses, or interpretation of data; in the writing of the manuscript, or in the decision to publish the results.

\section{Abbreviations}

$\begin{array}{ll}\text { EV } & \text { Extracellular vesicles } \\ \text { FSC } & \text { Forward scatter } \\ \text { FACS } & \text { Fluorescence-activated cell sorting } \\ \text { HSV-1 } & \text { Herpes simplex virus-1 } \\ \text { HCMV } & \text { Human cytomegalovirus } \\ \text { MLV } & \text { Murine leukemia virus } \\ \text { NFC } & \text { Nanoscale flow cytometry } \\ \text { NIEP } & \text { Non-infectious enveloped particle } \\ \text { PFU } & \text { Plaque-forming units } \\ \text { SSC } & \text { Sideward scatter } \\ \text { VLP } & \text { Virus-like-particle }\end{array}$

\section{References}

1. Talbot, P.; Almeida, J. D., Human cytomegalovirus: purification of enveloped virions and dense bodies. J Gen Virol 1977, 36, (2), 345-9.

2. Arakelyan, A.; Fitzgerald, W.; Margolis, L.; Grivel, J. C., Nanoparticle-based flow virometry for the analysis of individual virions. J Clin Invest 2013, 123, (9), 3716-27.

3. Zicari, S.; Arakelyan, A.; Fitzgerald, W.; Zaitseva, E.; Chernomordik, L. V.; Margolis, L.; Grivel, J. C., Evaluation of the maturation of individual Dengue virions with flow virometry. Virology 2016, 488, 207.

4. Landowski, M.; Dabundo, J.; Liu, Q.; Nicola, A. V.; Aguilar, H. C., Nipah virion entry kinetics, composition, and conformational changes determined by enzymatic virus-like particles and new flow virometry tools. J Virol 2014, 88, (24), 14197-206.

5. El Bilali, N.; Duron, J.; Gingras, D.; Lippe, R., Quantitative Evaluation of Protein Heterogeneity within Herpes Simplex Virus 1 Particles. J Virol 2017, 91, (10).

6. Renner, T. M.; Tang, V. A.; Burger, D.; Langlois, M. A., Intact Viral Particle Counts Measured by Flow Virometry Provide Insight into the Infectivity and Genome Packaging Efficiency of Moloney Murine Leukemia Virus. J Virol 2020, 94, (2). 
7. Vlasak, J.; Hoang, V. M.; Christanti, S.; Peluso, R.; Li, F.; Culp, T. D., Use of flow cytometry for characterization of human cytomegalovirus vaccine particles. Vaccine 2016, 34, (20), 2321-8.

8. $\quad$ Arakelyan, A.; Fitzgerald, W.; King, D. F.; Rogers, P.; Cheeseman, H. M.; Grivel, J. C.; Shattock, R. J.; Margolis, L., Flow virometry analysis of envelope glycoprotein conformations on individual HIV virions. Sci Rep 2017, 7, (1), 948.

9. Loret, S.; El Bilali, N.; Lippe, R., Analysis of herpes simplex virus type I nuclear particles by flow cytometry. Cytometry A 2012, 81, (11), 950-9.

10. Tang, V. A.; Renner, T. M.; Fritzsche, A. K.; Burger, D.; Langlois, M. A., Single-Particle Discrimination of Retroviruses from Extracellular Vesicles by Nanoscale Flow Cytometry. Sci Rep 2017, 7, (1), 17769.

11. Sandbaumhuter, M.; Dohner, K.; Schipke, J.; Binz, A.; Pohlmann, A.; Sodeik, B.; Bauerfeind, R., Cytosolic herpes simplex virus capsids not only require binding inner tegument protein pUL36 but also pUL37 for active transport prior to secondary envelopment. Cell Microbiol 2013, 15, (2), 248-69.

12. Bosse, J. B.; Hogue, I. B.; Feric, M.; Thiberge, S. Y.; Sodeik, B.; Brangwynne, C. P.; Enquist, L. W., Remodeling nuclear architecture allows efficient transport of herpesvirus capsids by diffusion. Proc Natl Acad Sci U S A 2015, 112, (42), E5725-33.

13. Dargan, D. J.; Patel, A. H.; Subak-Sharpe, J. H., PREPs: herpes simplex virus type 1-specific particles produced by infected cells when viral DNA replication is blocked. J Virol 1995, 69, (8), 4924-32.

14. Zhang, L.; Zhou, M.; Stanton, R.; Kamil, J.; Ryckman, B. J., Expression Levels of Glycoprotein O (gO) Vary between Strains of Human Cytomegalovirus, Influencing the Assembly of gH/gL Complexes and Virion Infectivity. J Virol 2018, 92, (15).

15. Hogue, I. B.; Bosse, J. B.; Engel, E. A.; Scherer, J.; Hu, J. R.; Del Rio, T.; Enquist, L. W., Fluorescent Protein Approaches in Alpha Herpesvirus Research. Viruses 2015, 7, (11), 5933-61.

16. Staropoli, I.; Dufloo, J.; Ducher, A.; Commere, P. H.; Sartori-Rupp, A.; Novault, S.; Bruel, T.; Lorin, V.; Mouquet, H.; Schwartz, O.; Casartelli, N., Flow Cytometry Analysis of HIV-1 Env Conformations at the Surface of Infected Cells and Virions: Role of Nef, CD4, and SERINC5. J Virol 2020, 94, (6).

17. Tang, V. A.; Renner, T. M.; Varette, O.; Le Boeuf, F.; Wang, J.; Diallo, J. S.; Bell, J. C.; Langlois, M. A., Single-particle characterization of oncolytic vaccinia virus by flow virometry. Vaccine 2016, 34, (42), 5082-5089.

18. Khalil, J. Y.; Langlois, T.; Andreani, J.; Sorraing, J. M.; Raoult, D.; Camoin, L.; La Scola, B., Flow Cytometry Sorting to Separate Viable Giant Viruses from Amoeba Co-culture Supernatants. Front Cell Infect Microbiol 2016, 6, 202.

19. Bonar, M. M.; Tilton, J. C., High sensitivity detection and sorting of infectious human immunodeficiency virus (HIV-1) particles by flow virometry. Virology 2017, 505, 80-90.

20. Gaudin, R.; Barteneva, N. S., Sorting of small infectious virus particles by flow virometry reveals distinct infectivity profiles. Nat Commun 2015, 6, 6022.

21. Heilingloh, C. S.; Krawczyk, A., Role of L-Particles during Herpes Simplex Virus Infection. Front Microbiol 2017, 8, 2565.

22. Bello-Morales, R.; Ripa, I.; Lopez-Guerrero, J. A., Extracellular Vesicles in Viral Spread and Antiviral Response. Viruses 2020, 12, (6).

23. Caobi, A.; Nair, M.; Raymond, A. D., Extracellular Vesicles in the Pathogenesis of Viral Infections in Humans. Viruses 2020, 12, (10).

24. Zicari, S.; Arakelyan, A.; Palomino, R. A. N.; Fitzgerald, W.; Vanpouille, C.; Lebedeva, A.; Schmitt, A.; Bomsel, M.; Britt, W.; Margolis, L., Human cytomegalovirus-infected cells release extracellular vesicles that carry viral surface proteins. Virology 2018, 524, 97-105. 
25. Turner, D. L.; Korneev, D. V.; Purdy, J. G.; de Marco, A.; Mathias, R. A., The host exosome pathway underpins biogenesis of the human cytomegalovirus virion. Elife 2020, 9.

26. Streck, N. T.; Zhao, Y.; Sundstrom, J. M.; Buchkovich, N. J., Human Cytomegalovirus Utilizes Extracellular Vesicles To Enhance Virus Spread. J Virol 2020, 94, (16).

27. Wittels, M.; Spear, P. G., Penetration of cells by herpes simplex virus does not require a low $\mathrm{pH}-$ dependent endocytic pathway. Virus Res 1991, 18, (2-3), 271-90.

28. Vanarsdall, A. L.; Johnson, D. C., Human cytomegalovirus entry into cells. Curr Opin Virol 2012, 2, (1), $37-42$.

29. Ricklefs, F. L.; Maire, C. L.; Reimer, R.; Duhrsen, L.; Kolbe, K.; Holz, M.; Schneider, E.; Rissiek, A.; Babayan, A.; Hille, C.; Pantel, K.; Krasemann, S.; Glatzel, M.; Heiland, D. H.; Flitsch, J.; Martens, T.; Schmidt, N. O.; Peine, S.; Breakefield, X. O.; Lawler, S.; Chiocca, E. A.; Fehse, B.; Giebel, B.; Gorgens, A.; Westphal, M.; Lamszus, K., Imaging flow cytometry facilitates multiparametric characterization of extracellular vesicles in malignant brain tumours. J Extracell Vesicles 2019, 8, (1), 1588555.

30. Nitta, N.; Sugimura, T.; Isozaki, A.; Mikami, H.; Hiraki, K.; Sakuma, S.; Iino, T.; Arai, F.; Endo, T.; Fujiwaki, Y.; Fukuzawa, H.; Hase, M.; Hayakawa, T.; Hiramatsu, K.; Hoshino, Y.; Inaba, M.; Ito, T.; Karakawa, H.; Kasai, Y.; Koizumi, K.; Lee, S.; Lei, C.; Li, M.; Maeno, T.; Matsusaka, S.; Murakami, D.; Nakagawa, A.; Oguchi, Y.; Oikawa, M.; Ota, T.; Shiba, K.; Shintaku, H.; Shirasaki, Y.; Suga, K.; Suzuki, Y.; Suzuki, N.; Tanaka, Y.; Tezuka, H.; Toyokawa, C.; Yalikun, Y.; Yamada, M.; Yamagishi, M.; Yamano, T.; Yasumoto, A.; Yatomi, Y.; Yazawa, M.; Di Carlo, D.; Hosokawa, Y.; Uemura, S.; Ozeki, Y.; Goda, K., Intelligent Image-Activated Cell Sorting. Cell 2018, 175, (1), 266-276 e13.

31. Armitage, J. D.; Tan, D. B. A.; Cha, L.; Clark, M.; Gray, E. S.; Fuller, K. A.; Moodley, Y. P., A standardised protocol for the evaluation of small extracellular vesicles in plasma by imaging flow cytometry. $J$ Immunol Methods 2019, 468, 61-66.

32. Sampaio, K. L.; Jahn, G.; Sinzger, C., Applications for a dual fluorescent human cytomegalovirus in the analysis of viral entry. Methods Mol Biol 2013, 1064, 201-9. 